\title{
Interval Type-2 Intuitionistic Fuzzy Logic System for Forecasting the Electricity Load
}

\author{
Imo Eyoh ${ }^{1}$, Jeremiah Eyoh ${ }^{2}$, Ini Umoeka ${ }^{3}$ \\ ${ }^{1,3}$ Department of Computer Science, University of Uyo, Uyo \\ Akwa Ibom State, Nigeria. \\ ${ }^{2}$ School of Electrical, Electronics and Systems Engineering \\ AVRRC Research Group, Loughborough University, U.K
}

\begin{abstract}
A study on load forecasting prediction is important for efficient management of users' demands for any utility such as electricity and gas. Many computational intelligence approaches have been adopted for electricity load forecasts. For instance, artificial neural networks, traditional fuzzy neural networks, support vector machines and other intelligent methods have been adopted in the literature. It is well known that load forecast is an important input for management decision systems in every power supply organization. Fuzzy logic systems (type-1 and type-2) have proven to be one of the effective tools for many load forecasting problems. In this study, interval type-2 intuitionistic fuzzy logic system equipped with membership functions, non-membership functions and hesitation indices is proposed for very short and short-term load forecasting for the first time. Gradient descent method is used to optimize the parameters of the developed model. The proposed model is compared with the traditional interval type-2 fuzzy logic and neural network systems. Experimental analyses reveal that the proposed model outperforms the two traditional systems in many load instances.
\end{abstract}

Key Words: Intuitionistic Fuzzy Set, Interval Type-2 Intuitionistic Fuzzy Set, Hesitation Index, Gradient Descent Back Propagation.

\section{INTRODUCTION}

Electricity load forecasting (ELF) has formed an important aspect in power generation, distribution and transmission sectors and has been one of the hot topics in recent years. The distribution of electricity load to industrial, residential and commercial consumers has been a challenging task in the power system and as such, providing adequate load forecast is important for efficient and reliable functioning of the system. Load forecasting has provided support in the power system decision making in the form of demand scheduling, supply planning, generation reserve and more [1]. Load forecasting applications may be classified as [2], [3].

- Very short-term load forecasting (VSTLF) which involves forecasting for few minutes to few hours' time scale. - Short-term load forecasting (STLF) involves prediction of electricity demand over hourly/half-hourly intervals up to few days ahead. STLF has been one of the active ingredients in energy management systems (EMS) and is known to assist in the decisionmaking process of every power system [4].

- Medium term load forecasting (MTLF) ranges from one week up to a few months and

- Long term load forecasting (LTLF) which can span up to a year and above [5]. In this study, analysis is done based on VSTLF and STLF.

Many approaches have been utilized for load forecasting. Torrini et al., [6] have pointed out four of such approaches to include a) statistical models which are based on linear regression function capable of predicting stationary time series [7]. However, 
statistical models often perform poorly and their prediction accuracies found to be insufficient [8], b) computational intelligence models which include among others fuzzy logic and neural network, c) hybrid models and d) those models used for judgmental forecasting. The statistical and computational intelligence models are widely adopted for load forecasting [7]. For instance, Chen et al., [9] proposed a least square support vector machine (LSSVM) with fuzzy time series and global harmony search algorithm for electric load forecasting. Whilst LSSVM was used to analyze the time series, the global harmony search algorithm was adopted to optimize the parameters of the model. The proposed model was shown to provide more accurate results than other comparative models such as autoregressive integrated moving average, and other LSSVM hybrid models. Sadaei et al., [4] proposed a combination of seasonal auto regressive fractionally integrated moving average (SARFIMA) and fuzzy time series (FTS) for short term load forecasting. The parameters of the model are updated using particle swarm optimization algorithm. The authors concluded that the hybrid approach outperformed many existing methods for load forecasting in terms of prediction error. Amin and Hoque [10] carried out a comparative load forecasting analysis between auto-regressive integrated moving average (ARIMA) and support vector machine (SVM). Their analyses showed that both models perform differently under certain load condition. Whilst ARIMA outperforms SVM under linear load type, SVM performs better than ARIMA under non-linear load conditions. Haq and $\mathrm{Ni}$ [7] proposed a hybrid model for short term electricity forecasting based on signal decomposition and correlation analysis approaches. The proposed model was compared with some traditional approaches and found to perform better in terms of prediction errors. Ma et al., [11] proposed a STLF using isolation forest (iForest) algorithm and long short term memory (LSTM) neural network. Comparison of the proposed model with the traditional LSTM and iForest backpropagation (BP) showed that the proposed model exhibited best load forecasting performance. Ouyang et al., [12] presented a STLF using copula model and deep belief network. Data for the experiments were collected from Texas and Arkansas, United States. Performance comparison was made between the proposed approach and artificial neural network (ANN), SVM, extreme learning machine and traditional deep belief network; and the proposed model was found to outperform these existing models. Yan et al., [13] proposed a STLF of smart grid based on load spatial-temporal distribution (LSTD) with LSTM. Two cases BP-, SVM- under LSTD are set up to compare with the proposed LSTM version. Analysis of the study revealed that LSTM for STLF based on LSTD provided improved performance in comparison to BP and SVM. Chen et al., [14] and [15] presented a STLF with deep neural network. In Ryu et al., [16], demand side STLF is proposed based on deep neural network (DNN). Demand side hourly load data for the experiment was obtained from Korea Electric Power Corporation (KEPCO) which included individual customers electricity consumption data and regional meteorological elements.

However, because load data is uncertain, statistical models and some computational approaches such as neural networks (NN), SVM, genetic algorithm and others may not be able to cope with these imprecise and vague information as standalone systems. According to [12], the statistical approaches based on simple regression functions may not be effective in modeling nonlinear characteristics. According to Haq et al., [7], load demand generally is non-stationary and exhibits non-linear features. Although ANN works well with nonlinear systems, they are often found to converge slowly and may get trapped in local minimum. Another difficulty encountered with neural network however, is that its prediction dynamics cannot be explained and as such is incomprehensible to the human users who require understanding of how the conclusion is reached especially in real world applications. In this way, integration of fuzzy logic methodologies has been adopted. A technology that has therefore boosted and paved the way for effective utilization of ANN is the marriage of fuzzy logic theory and ANN. These combinations have been adopted by researchers to solve many real-world problems in diverse domains and magnitude. In the literature, fuzzy logic and ANN are found to be universal approximators and can approximate any nonlinear relationship to any arbitrary accuracy [17]. Whilst fuzzy logic handles the reasoning with imprecise and uncertain data, ANN handles the learning and generalization ability. According to Khosravi et al., [17] the integration of fuzzy logic systems and artificial neural networks provides an excellent alternative for prediction, modelling and forecasting. Fuzzy set (FS) of type- 1 introduced by Zadeh [18] has been used extensively for modelling uncertainty in many applications such as load forecasting with encouraging results. For example, Torrini et al., [6] proposed a long-term forecast for electric consumption in Brazil based on type-1 fuzzy logic system. The fuzzy logic model was found to produce accurate results when compared with official projections. Akarslan and Hocaoglu [3] employed adaptive neuro-fuzzy inference system (ANFIS) for STLF with data collected from Cay vocational high school campus area of Afyon Kotatepe University. The authors concluded that ANFIS approach gave good forecasting results even though comparison was not made with other existing approaches. Other studies integrating fuzzy logic for load forecasting include [19], [20], [21], [22]. However, FLSs of type-1 such as ANFIS are described only by membership functions where the uncertainty disappears immediately the output value is chosen. This deficiency in type-1 FS led to the introduction of type-2 FS in [23], which is an extension of the type-1 FS. Since then, there has been an upsurge in the use of type-2 FS to solve many real-world problems including load forecasting. For instance, Khosravi et al., [17] proposed a TSK-IT2FLS for STLF. The aim was to manage uncertainty in the load data with improved prediction. Analysis of findings showed that IT2FLS-STLF outperformed its type-1, and traditional neural networks in terms of prediction accuracy. Umoh et al., [24] applied IT2F neural network (IT2FNN) for short term electric load forecasting using historical data obtained from Power Holding Company of Nigeria (PHCN) and Nigeria Meteorological Agency (NMA). The experimental studies showed that the IT2FNN outperformed type-1 FLS. Type-2 FS are 
found to outperform type-1 FS in many applications because type-2 FSs have membership functions that are both type-1 FS (fuzzy membership functions). Type-2 FS is a 3-dimensional FS and may be represented either as general type-2 FS (GT2FS) with different weights on the third dimension or interval type-2 FS (IT2FS) where the third dimension takes the value 1 (equally weighted). Type-2 FS with fuzzy membership functions comes with additional design degrees of freedom which gives it an edge over the type-1 FS with the possibility to directly model uncertainties [25]. Both the type-1 and type-2 forms of the traditional FS are well defined by their membership functions only. In other words, to obtain the non-membership function of a traditional FS requires obtaining the complement of the membership functions. This may not generally fit into real world situations. Another widely used extension of FS is the intuitionistic FS (IFS) introduced by Atanassov in 1986 [26]. IFS, unlike the classical FS has both membership and non-membership functions that are independent of each other. One of the unique things about IFS is the presence of the hesitation degree (index). As obtained with the type-1 FS, the IFS with singular membership and non-membership functions may not adequately model uncertainty in many real-world applications. On this premise, Eyoh et al., [27] proposed a rule-based interval type-2 intuitionistic fuzzy logic system (IT2IFLS) with fuzzy membership and non-membership functions and applied it to solve nonlinear system predictions. Many other researches have been conducted successfully using IT2IFLS. For instance, IT2IFS have been utilized for identification and prediction problems [28], [29], transportation problem [30], clustering [31], time series [32] [33], image segmentation [34], regression [35], [36], [25] with promising results. In this study, IT2IFLS is adopted for both very short-term load forecasting (IT2IFLS-VSTLF) and short-term load forecasting (IT2IFLS-STLF) for the first time. The major contribution of this research is the forecasting of electricity load for the first-time using membership and nonmembership functions that are themselves fuzzy. Another important contribution is the integration of hesitation index in the forecasting apparatus which is often neglected in load forecasting analysis. The hesitation index is a part of everyday language representation and tends to bring experimental analysis closer to natural language in the best possible way. It is interesting therefore to study the concept of load forecasting in the context of a hesitation enabled-fuzzy logic of type-2 and compare performances.

To the best knowledge of the authors, IT2IFLS with membership functions, non-membership functions and hesitation indices has never been adopted for the forecasting of electricity load demand. The remaining part of the paper are as follows: Section 2 discusses intuitionistic fuzzy set (type-1 and type-2), interval type-2 intuitionistic fuzzy logic system and parameter update rule for IT2IFLS. In Section 3, the dataset used in the analysis is described with performance evaluation in Section 4. The study is concluded in Section 5.

\section{INTUITIONISTIC FUZZY SET}

Definition 1: An intuitionistic fuzzy set is identified by its membership function and non-membership function: $G^{*}=$ $\left\{\left\langle x, \mu_{G^{*}}(x), v_{G^{*}}(x)\right\rangle \mid x \in X\right\}$ with hesitation index $(\pi)$ such that $\mu_{G^{*}}(x)+v_{G^{*}}(x) \leq 1[26]$.

where the function:

$\mu_{G^{*}}(x): X \rightarrow[0,1]$ is the degree that $x \in X$ and this represents the membership function of element $x$. and the function:

$v_{G^{*}}(x): X \rightarrow[0,1]$ is the degree that $x$ is not in $X$ and this represents the non-membership function of element $x$. For IFS, $0 \leq \mu_{G^{*}}(x)+v_{G^{*}}(x) \leq 1$. The hesitation index of element, $x$, defined as $\pi(x)$ is such that $\pi_{G^{*}}(x)=1-\left(\mu_{G^{*}}(x)+v_{G^{*}}(x)\right)$. Obviously, $0 \leq \pi_{G^{*}}(x) \leq 1$. This measures the degree of hesitancy of the expert in the specification of the belongingness of the element $x$ to the IFS $G^{*}$.

\subsection{Interval Type-2 Intuitionistic Fuzzy Set (IT2IFS)}

Definition 2: An IT2IFS, $\tilde{G}^{*}$ is characterized by membership functions (lower and upper) and non-membership functions (lower and upper) defined as $\left\{\bar{\mu}_{\tilde{G}^{*}}(\mathrm{x}), \underline{\mu}_{\tilde{G}^{*}}(\mathrm{x})\right\}$ and $\left\{\bar{v}_{\tilde{G}^{*}}(\mathrm{x}), \underline{v}_{\tilde{G}^{*}}(\mathrm{x})\right\}$ respectively for all $x \in X$ with constraints: $0 \leq \bar{\mu}_{\tilde{G}^{*}}(\mathrm{x}),+\underline{v}_{\tilde{G}^{*}}(\mathrm{x}) \leq$ 1 and $0 \leq \underline{\mu}_{\tilde{G}^{*}}(\mathrm{x})+\bar{v}_{\tilde{G}^{*}}(\mathrm{x}), \leq 1$ [31]. Both the membership and non-membership functions of IT2IFLS can be represented on a two-dimensional plane which makes computation easier and manageable. An IT2IFS is defined by two type-1 fuzzy membership functions and two type-1 non-membership functions, hence two footprints of uncertainty (FOU) suffice, one for membership function and the other for non-membership function [27] (see Fig. 1) as shown below:

$$
\begin{aligned}
& \operatorname{FOU}_{\mu}\left(\tilde{G}^{*}\right)=\bigcup_{\forall x \in X}\left[\underline{\mu}_{\tilde{G} *}(x), \bar{\mu}_{\tilde{G} *}(x)\right] \\
& F O U_{v}\left(\tilde{G}^{*}\right)=\bigcup_{\forall x \in X}\left[\underline{v}_{\tilde{G} *}(x), \bar{v}_{\tilde{G} *}(x)\right]
\end{aligned}
$$




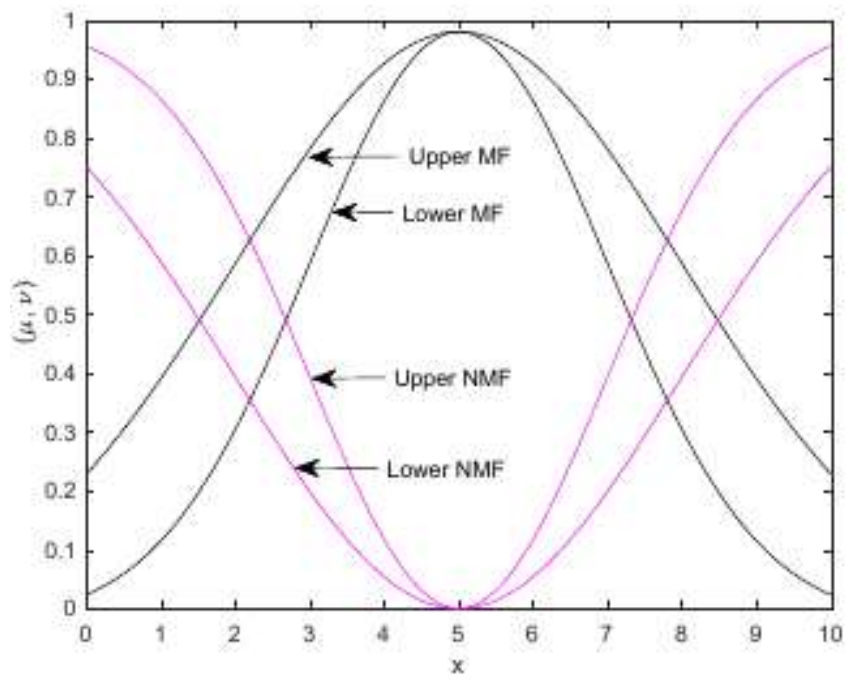

Figure 1: Plot of IT2IFS [27]

Equations (1) and (2) represent FOUs for membership and non-membership functions respectively.

Two IF-indices used in this study are the IF-index of center and IF-index of variance [27] as below:

$$
\begin{aligned}
& \pi_{\mathrm{c}}(\mathrm{x})=\max \left(0,\left(1-\left(\mu_{\tilde{G}^{*}}(\mathrm{x})+v_{\tilde{G}^{*}}(\mathrm{x})\right)\right)\right) \\
& \bar{\pi}_{\mathrm{var}}(\mathrm{x})=\max \left(0,\left(1-\left(\bar{\mu}_{\tilde{G}^{*}}(\mathrm{x})+\underline{v}_{\tilde{G}^{*}}(\mathrm{x})\right)\right)\right) \\
& \underline{\pi}_{\mathrm{var}}(\mathrm{x})=\max \left(0,\left(1-\left(\underline{\mu}_{\tilde{G}^{*}}(\mathrm{x})+\bar{v}_{\tilde{G}^{*}}(\mathrm{x})\right)\right)\right)
\end{aligned}
$$

\subsection{Interval Type-2 Intuitionistic Fuzzy Logic System}

As with the traditional FLS, the IT2IFLS consists of four major computing blocks namely; intuitionistic (fuzzifier, rule base, inference engine and output processing (type reducer + defuzziffier)). Working with any fuzzy system involves first reducing the external inputs into fuzzy inputs. This is achieved using the fuzzifier. The inputs are translated into IT2IFS using intuitionistic fuzzifier. Here Gaussian membership and non-membership functions are adopted. The Gaussian function is well known for its smoothness and differentiability at all points and has been a popularly used function in fuzzy system applications. The intuitionistic Gaussian membership and non-membership functions are defined as follows:

$$
\begin{gathered}
\bar{\mu}_{i k}\left(x_{i}\right)=\exp \left(-\frac{\left(x_{i}-c_{i k}\right)^{2}}{2 \sigma_{2, i k}^{2}}\right) *\left(1-\pi_{c, i k}\left(x_{i}\right)\right) \\
\underline{\mu}_{i k}\left(x_{i}\right)=\exp \left(-\frac{\left(x_{i}-c_{i k}\right)^{2}}{2 \underline{\sigma}_{1, i k}^{2}}\right) *\left(1-\pi_{c, i k}\left(x_{i}\right)\right) \\
\bar{v}_{i k}\left(x_{i}\right)=\left(1-\underline{\pi}_{v a r, i k}\left(x_{i}\right)\right)-\bar{\mu}_{i k}\left(x_{i}\right) \\
\bar{v}_{i k}\left(\left(x_{i}\right)=\left(1-\bar{\pi}_{v a r, i k}\left(x_{i}\right)\right)-\underline{\mu}_{i k}\left(x_{i}\right)\right.
\end{gathered}
$$

where $\pi_{c}$ is the hesitation degree for the center and $\pi_{v a r}$ is the hesitation degree for the variance. The premise parameters $c, \sigma_{2}, \sigma_{1}$ together with $\pi_{c}$ and $\pi_{v a r}$ are used in the antecedent for the definition of membership and non-membership functions of IT2IFS. The intuitionistic fuzzy inputs are further combined to generate rules in the rule base which activate the inference engine. IT2IFLS-TSK rule may be represented as:

$R k: I F x_{1}$ is $\tilde{G}_{1 k}^{*}$ and $x_{2}$ is $\tilde{G}_{2 k}^{*}$ and $\cdots$ and $x_{n}$ is $\tilde{G}_{n k}^{*}$ THEN $p_{k}=w_{1 k} x_{1}+w_{2 k} x_{2}+\cdots+w_{n k} x_{n}+b_{k}$

where $\tilde{G}_{i k}^{*}$ are IT2IFS and $p_{k}$ is the output of the $k_{t h}$ rule. The generated intuitionistic fuzzy output sets are then type-reduced and defuzzified to obtain the final crisp output of a T2IFLS. 


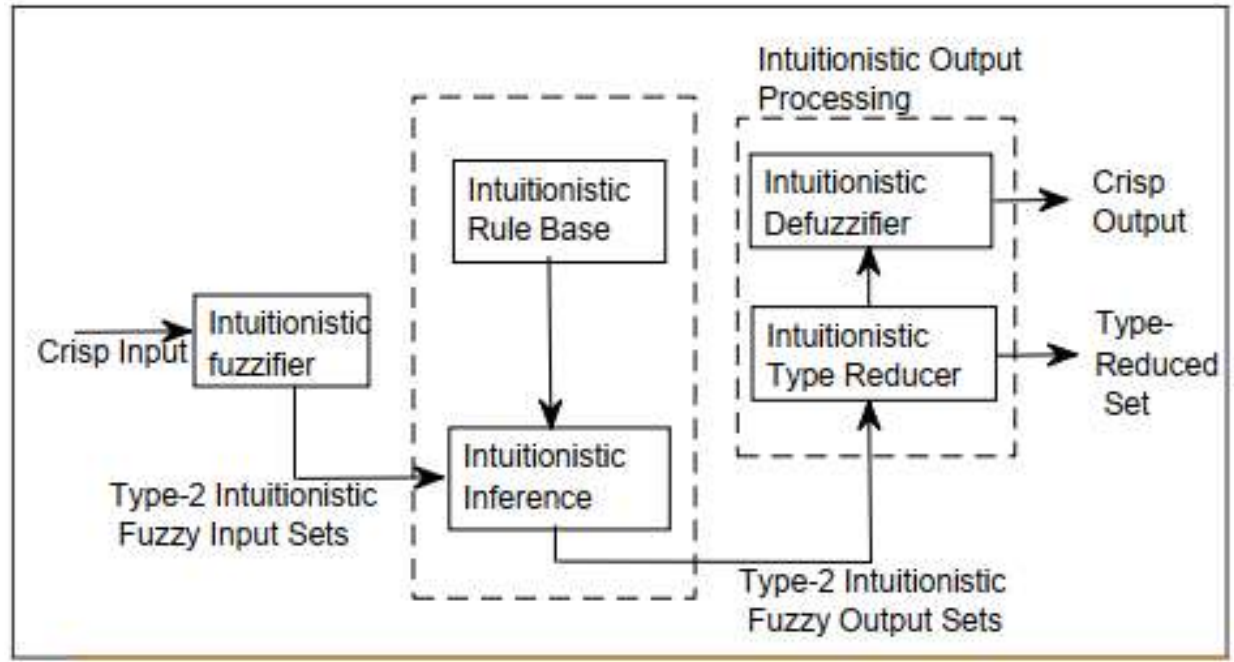

Figure 2: IT2IFLS [36]

Often the Karnik-Mendel iterative type-reduction procedure is employed to reduce T2FS to T1FS. However, going through the type reduction procedure is very tedious and computationally expensive [37]. Many algorithms have been developed which circumvent the type-reduction procedure and directly compute the output of an IT2FLS. Among such approaches are BegianMelek-Mendel (BMM) output procedure [38], Nie-Tan [39], Wu and Tan [40] to mention but a few. This study adopts and modified the direct output computation in [38] for IT2IFLS as below:

$$
p=\frac{(1-\beta) \sum_{k=1}^{M}\left(\underline{f_{k}^{\mu}}+\overline{f_{k}^{\mu}}\right) p_{k}^{\mu}}{\sum_{k=1}^{M} \underline{f_{k}^{\mu}}+\sum_{k=1}^{M} \overline{f_{k}^{\mu}}}+\frac{\beta \sum_{k=1}^{M}\left(\underline{f_{k}^{v}}+\overline{f_{k}^{v}}\right) p_{k}^{v}}{\sum_{k=1}^{M} \underline{f_{k}^{v}}+\sum_{k=1}^{M} \overline{f_{k}^{v}}}
$$

With "prod" t-norm, the following are obtained for $\underline{f_{k}^{\mu}}, \overline{f_{k}^{\mu}}, \underline{f_{k}^{v}}$ and $\overline{f_{k}^{v}}$.

$$
\begin{gathered}
\frac{f_{k}^{\mu}}{\underline{-}}=\underline{\mu}_{\tilde{G}_{1 k}^{*}}\left(x_{1}\right) * \underline{\mu}_{\tilde{G}_{2 k}^{*}}\left(x_{2}\right) * \ldots * \underline{\mu}_{\tilde{G}_{n k}^{*}}\left(x_{n}\right) \\
\overline{f_{k}^{\mu}}=\bar{\mu}_{\tilde{G}_{1 k}^{*}}\left(x_{1}\right) * \bar{\mu}_{\tilde{G}_{2 k}^{*}}\left(x_{2}\right) * \ldots * \bar{\mu}_{\tilde{G}_{n k}^{*}}\left(x_{n}\right) \\
\overline{f_{k}^{v}}=\bar{v}_{\tilde{G}_{1 k}^{*}}\left(x_{1}\right) * \bar{v}_{\tilde{G}_{2 k}^{*}}\left(x_{2}\right) * \ldots * \bar{v}_{\tilde{G}_{n k}^{*}}\left(x_{n}\right) \\
\underline{f_{k}^{v}}=\underline{v}_{\tilde{G}_{1 k}^{*}}\left(x_{1}\right) * \underline{v}_{\tilde{G}_{2 k}^{*}}\left(x_{2}\right) * \ldots * \underline{v}_{\tilde{G}_{n k}^{*}}\left(x_{n}\right)
\end{gathered}
$$

which represent the firing magnitude for lower membership, upper membership, lower non-membership and upper nonmembership functions respectively. The parameter $\beta$ in (11) is defined by the user to scale the membership and non-membership contributions in the final output and is defined such that, $0 \leq \beta \leq 1$. Obviously, if $\beta=0$, the outputs of the IT2IFLS is contributed only by the membership function and if $\beta=1$, output is formed by the non-membership function only, otherwise both functions contribute to the final output.

\subsection{IT2IFLS Parameter Update using Gradient Descent}

The IT2IFLS-VSTLS and STLF presented here are minimization problems with the aim of minimizing the cost function which is defined as in (16) for a single output.

$$
E=\frac{1}{2}\left(p^{a}-p\right)^{2}
$$

where $p^{a}$ represents the actual output and $p$ is the predicted output of IT2IFLS. The update rules based on GD are represented as in (17) to (21):

$$
\begin{aligned}
w_{i k}(t+1)=w_{i k}(t) & -\gamma \frac{\partial E}{\partial w_{i k}} \\
b_{k}(t+1) & =b_{k}(t)-\gamma \frac{\partial E}{\partial b_{k}}
\end{aligned}
$$


International Journal of Advances in Scientific Research and Engineering (ijasre), Vol 6 (10), October -2020

$$
\begin{aligned}
& c_{i k}(t+1)=c_{i k}(t)-\gamma \frac{\partial E}{\partial c_{i k}} \\
& \sigma_{1, i k}(t+1)=\sigma_{1, i k}(t)-\gamma \frac{\partial E}{\partial \sigma_{1, i k}} \\
& \sigma_{2, i k}(t+1)=\sigma_{2, i k}(t)-\gamma \frac{\partial E}{\partial \sigma_{2, i k}}
\end{aligned}
$$

where $\gamma$ is the learning rate, $w ; b$ are the parameters of the consequent parts and $c ; \sigma 1 ; \sigma 2$ are parameters of the antecedent parts. The adjustments of the consequent parameters of IT2IFLS are as follows:

$$
\begin{aligned}
& \frac{\partial E}{\partial w_{i k}}=\frac{\partial E}{\partial p} \frac{\partial p}{\partial p_{k}} \frac{\partial p_{k}}{\partial w_{i k}}=\sum_{k=1}^{M} \frac{\partial E}{\partial p}\left[\frac{\partial p}{\partial p_{k}^{\mu}} \frac{\partial p_{k}^{\mu}}{\partial w_{i k}}+\frac{\partial p}{\partial p_{k}^{v}} \frac{\partial p_{k}^{v}}{\partial w_{i k}}\right] \\
& =\left(p^{a}(t)-p(t)\right) *\left[(1-\beta)\left(\frac{f_{k}^{\mu}}{\sum_{k=1}^{M} \underline{f}_{k}^{\mu}+\sum_{k=1}^{M} \bar{f}_{k}^{\mu}}+\frac{\bar{f}_{k}^{\mu}}{\sum_{k=1}^{M} f_{k}^{\mu}+\sum_{k=1}^{M} \bar{f}_{k}^{\mu}}\right)+\beta\left(\frac{\underline{f}_{k}^{v}}{\sum_{k=1}^{M} f_{k}^{v}+\sum_{k=1}^{M} \bar{f}_{k}^{v}}+\frac{f_{k}^{v}}{\sum_{k=1}^{M} \underline{f}_{k}^{v}+\sum_{k=1}^{M} \bar{f}_{k}^{v}}\right)\right] * x_{i} \\
& \frac{\partial E}{\partial b_{k}}=\frac{\partial E}{\partial p} \frac{\partial p}{\partial p_{k}} \frac{\partial p_{k}}{\partial b_{k}}=\sum_{k=1}^{M} \frac{\partial E}{\partial p}\left[\frac{\partial p}{\partial p_{k}^{\mu}} \frac{\partial p_{k}^{\mu}}{\partial b_{k}}+\frac{\partial p}{\partial p_{k}^{v}} \frac{\partial p_{k}^{v}}{\partial b_{k}}\right] \\
& =\left(p^{a}(t)-p(t)\right) *\left[(1-\beta)\left(\frac{f_{k}^{\mu}}{\sum_{k=1}^{M} \underline{f}_{k}^{\mu}+\sum_{k=1}^{M} \bar{f}_{k}^{\mu}}+\frac{\bar{f}_{k}^{\mu}}{\sum_{k=1}^{M} f_{k}^{\mu}+\sum_{k=1}^{M} \bar{f}_{k}^{\mu}}\right)+\beta\left(\frac{f_{k}^{v}}{\sum_{k=1}^{M} f_{k}^{v}+\sum_{k=1}^{M} \bar{f}_{k}^{v}}+\frac{\bar{f}_{k}^{v}}{\sum_{k=1}^{M} \underline{f}_{k}^{v}+\sum_{k=1}^{M} \bar{f}_{k}^{v}}\right)\right] * 1
\end{aligned}
$$

where $p_{k}$ is the output of the $k_{t h}$ rule. The updates for the center and standard deviations of the IT2IFLS are computed as follows:

$$
\begin{aligned}
& \frac{\partial E}{\partial c_{i k}}=\sum_{k=1}^{M} \frac{\partial E}{\partial p}\left[\frac{\partial p}{\partial f_{k}^{\mu}} \frac{\partial f_{k}^{\mu}}{\partial \underline{\mu}_{i k}} \frac{\partial \underline{\mu}_{i k}}{\partial c_{i k}}+\frac{\partial p}{\partial \bar{f}_{k}^{\mu}} \frac{\partial \bar{f}_{k}^{\mu}}{\partial \bar{\mu}_{i k}} \frac{\partial \bar{\mu}_{i k}}{\partial c_{i k}}+\frac{\partial p}{\partial \underline{f}_{k}^{v}} \frac{\partial \underline{f}_{k}^{v}}{\partial \underline{v}_{i k}} \frac{\partial \underline{v}_{i k}}{\partial c_{i k}}+\frac{\partial p}{\partial \bar{f}_{k}^{v}} \frac{\partial \bar{f}_{k}^{v}}{\partial \bar{v}_{i k}} \frac{\partial \bar{v}_{i k}}{\partial c_{i k}}\right] \\
& \frac{\partial E}{\partial \sigma_{1, i k}}=\sum_{k=1}^{M} \frac{\partial E}{\partial p}\left[\frac{\partial p}{\partial \underline{f}_{k}^{\mu}} \frac{\partial \underline{f}_{k}^{\mu}}{\partial \underline{\mu}_{i k}} \frac{\partial \underline{\mu}_{i k}}{\partial \sigma_{1, i k}}+\frac{\partial p}{\partial \bar{f}_{k}^{v}} \frac{\partial \bar{f}_{k}^{v}}{\partial \bar{v}_{i k}} \frac{\partial \bar{v}_{i k}}{\partial \sigma_{1, i k}}\right] \\
& \frac{\partial E}{\partial \sigma_{2, i k}}=\sum_{k=1}^{M} \frac{\partial E}{\partial p}\left[\frac{\partial p}{\partial \bar{f}_{k}^{\mu}} \frac{\partial \bar{f}_{k}^{\mu}}{\partial \bar{\mu}_{i k}} \frac{\partial \bar{\mu}_{i k}}{\partial \sigma_{2, i k}}+\frac{\partial p}{\partial \underline{f}_{k}^{v}} \frac{\partial \underline{f}_{k}^{v}}{\partial \underline{v}_{i k}} \frac{\partial \underline{v}_{i k}}{\partial \sigma_{2, i k}}\right]
\end{aligned}
$$

where:

$$
\frac{\partial p}{\partial f_{k}^{\mu}}=\frac{\partial p}{\partial \bar{f}_{k}^{\mu}}=(1-\beta)\left[\frac{p_{k}^{\mu}}{\sum_{k=1}^{M} f_{k}^{\mu}+\sum_{k=1}^{M} \bar{f}_{k}^{\mu}}-\frac{p^{\mu}}{\sum_{k=1}^{M} f_{k}^{\mu}+\sum_{k=1}^{M} \bar{f}_{k}^{\mu}}\right]
$$

where:

$$
\begin{gathered}
p^{\mu}=\frac{\sum_{k=1}^{M}\left(f_{k}^{\mu}+\bar{f}_{k}^{\mu}\right) * p_{k}^{\mu}}{\sum_{k=1}^{M} \underline{f}_{k}^{\mu}+\sum_{k=1}^{M} \bar{f}_{k}^{\mu}} \\
\frac{\partial p}{\partial f_{k}^{v}}=\frac{\partial p}{\partial \bar{f}_{k}^{v}}=\beta\left[\frac{p_{k}^{v}}{\sum_{k=1}^{M} \underline{f}_{k}^{v}+\sum_{k=1}^{M} \bar{f}_{k}^{v}}-\frac{p^{v}}{\sum_{k=1}^{M} \underline{f}_{k}^{v}+\sum_{k=1}^{M} \bar{f}_{k}^{v}}\right]
\end{gathered}
$$

where:

$$
p^{v}=\frac{\sum_{k=1}^{M}\left(f_{k}^{v}+\bar{f}_{k}^{v}\right) * p_{k}^{v}}{\sum_{k=1}^{M} \underline{f}_{k}^{v}+\sum_{k=1}^{M} \bar{f}_{k}^{v}}
$$


The derivatives for the non-membership functions are $\frac{\partial \underline{v}_{i k}}{\partial c_{i k}}=-\frac{\partial \underline{\mu_{i k}\left(x_{i}\right)}}{\partial c_{i k}}, \frac{\partial \bar{v}_{i k}}{\partial c_{i k}}=-\frac{\partial \bar{\mu}_{i k}\left(x_{i}\right)}{\partial c_{i k}}, \frac{\partial \bar{v}_{i k}}{\partial \sigma_{1, i k}}=-\frac{\partial \bar{\mu}_{i k}\left(x_{i}\right)}{\partial \sigma_{1, i k}}$ and $\frac{\partial \underline{v}_{i k}}{\partial \sigma_{2, i k}}=$ $-\frac{\partial \bar{\mu}_{i k}\left(x_{i}\right)}{\partial \sigma_{2, i k}}$. Then,

$$
\frac{\partial \underline{f}_{k}^{\mu}}{\partial \underline{\mu}_{i k}}=\prod_{j=1, j \neq i}^{M 1} \underline{\mu}_{j k}, \frac{\partial \bar{f}_{k}^{\mu}}{\partial \bar{\mu}_{i k}}=\prod_{j=1, j \neq i}^{M 1} \bar{\mu}_{j k}, \frac{\partial \underline{f}_{k}^{v}}{\partial \underline{v}_{i k}}=\prod_{j=1, j \neq i}^{M 1} \underline{v}_{j k} \text { and } \frac{\partial \bar{f}_{k}^{v}}{\partial \bar{v}_{i k}}=\prod_{j=1, j \neq i}^{M 1} \bar{v}_{j k},
$$

The parameter, $\beta$, is adjusted as follows:

$$
\frac{\partial E}{\partial \beta}=\frac{\partial E}{\partial p} \frac{\partial p}{\partial \beta}=\left(p^{a}-p\right)\left(p^{v}-p^{\mu}\right)
$$

\section{DATASET DESCRIPTION}

The dataset for the analysis is obtained from Australia's National Electricity Market (NEM) [41] which comprises five regions namely: New South Wales (NSW), Victoria (VIC), Queensland (QL), Tasmania (TAS) and South Australia (SA) electricity load. The data collected range from 1st of January to $31^{\text {st }}$ of December, 2018 on half hourly basis. There are four seasons in Australia namely; 1) Summer which falls between December and February, 2) Autumn which is between March and May, 3) Winter which falls between June and August and 4) Spring which is between September to November. The data is collected for each region and partitioned into the four seasons. Shown in Figures 3 to 7 are boxplots of the monthly load demand for each region. The boxplots give a holistic overview of the load demand in the different regions. As shown in the figures, the load demand increases significantly between the months of June and August in most of the regions which is the winter period in Australia. For the analysis of the load demand, the electricity load is modelled as a time series. In order to maintain an up-to-date forecasting, the current value of the time series is used as part of the input vector. By using the input generating vector: $[x(t) ; x(t-1) ; \ldots ; x(t-$ $q+1)$ ], where $q$ is the input size with $t \geq q$, this study employs four previous loads and current value as inputs into the model in order to predict one-step ahead of the time series on a half-hourly basis denoted by $x(t+1)$ in the first example. The previous values help to keep track of the trend in the series. Thus, the VSTLF is a half-hourly prediction. The second instance of the forecasting is on a short term (one-day) forecasting horizon (STLF). Thus, with the same input vector, the output vector becomes $x(t+48)$ equivalent to 24 hours. Before fuzzification procedure, the data are normalized using the min-max normalization as shown in (35).

$$
x_{\text {new }}=\frac{x(i)-\min (x)}{\max (x)-\min (x)}
$$

where $x(i),(i=1,2, \ldots, N)$ is the set of time series with $N$ instances, $\min (x)$ and $\max (x)$ are the minimum and maximum values of column data respectively. The generated dataset, $x_{\text {new }}$ is the normalized data fed into the IT2IFLS. With the min-max normalization, the features are scaled to lie in a narrow range between 0 and 1. IT2IFLS is an integration of type- 2 intuitionistic fuzzy logic and neural network. Literature shows that neural networks work best when the features are scaled to a small range between 0 and 1. As shown in Figures 3 to 7, the values of the load range from 0 to tens of thousands. Normalizing the values ensures that such large values do not overshadow the smaller values, leading to poor performance. Figure 8 shows the flowchart of the IT2IFLS load forecast process.

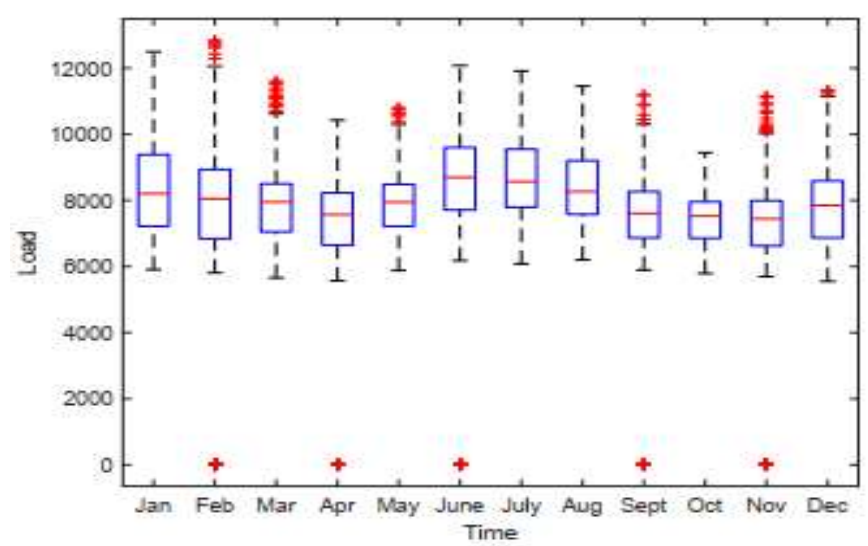

Figure 3. NSW load demand

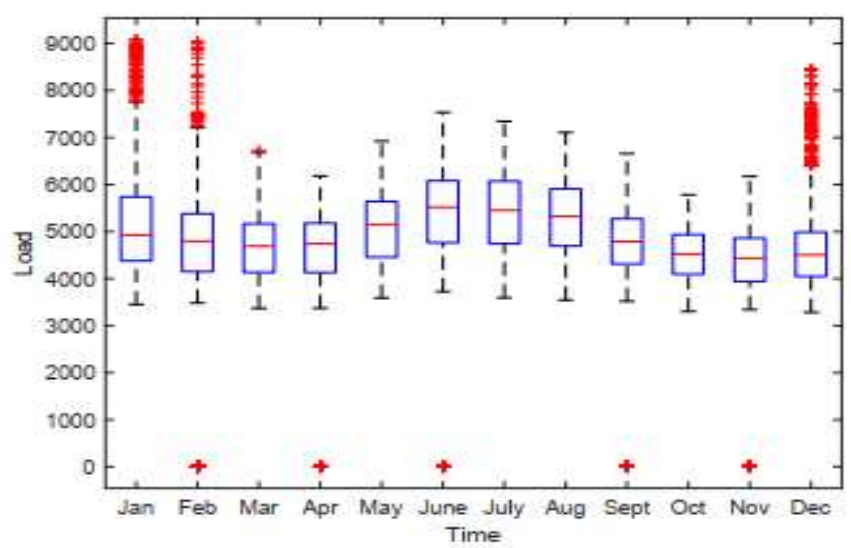

Figure 4. Victoria load demand 
International Journal of Advances in Scientific Research and Engineering (ijasre), Vol 6 (10), October -2020

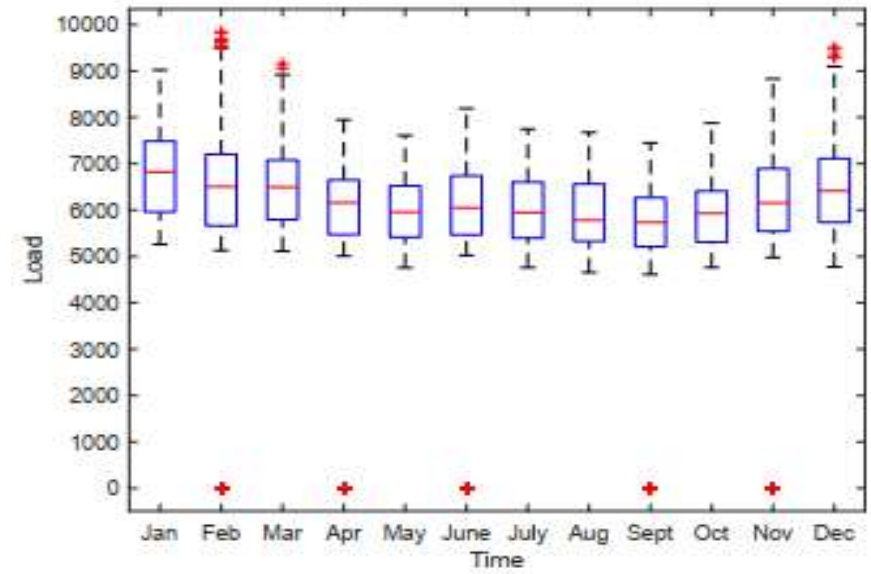

Figure 5. QL load demand

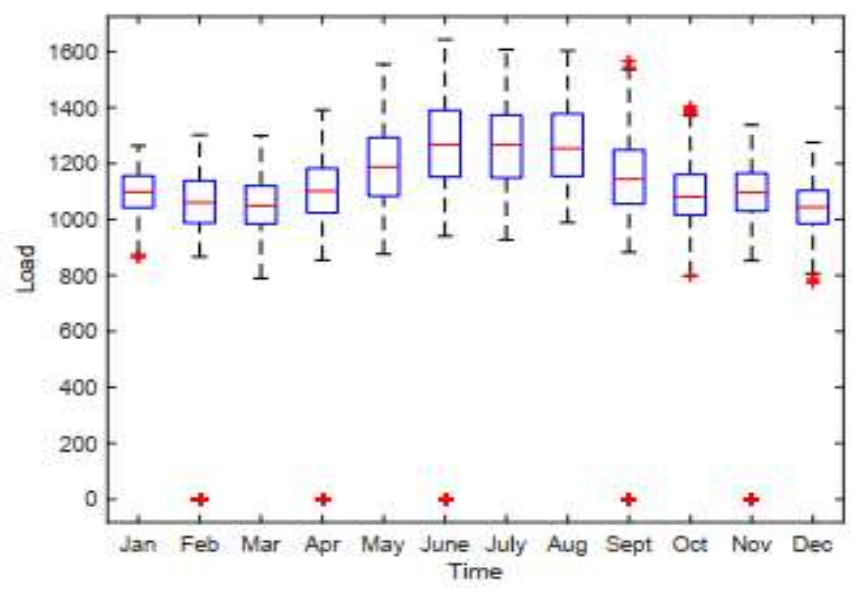

Figure 6. Tasmania load demand

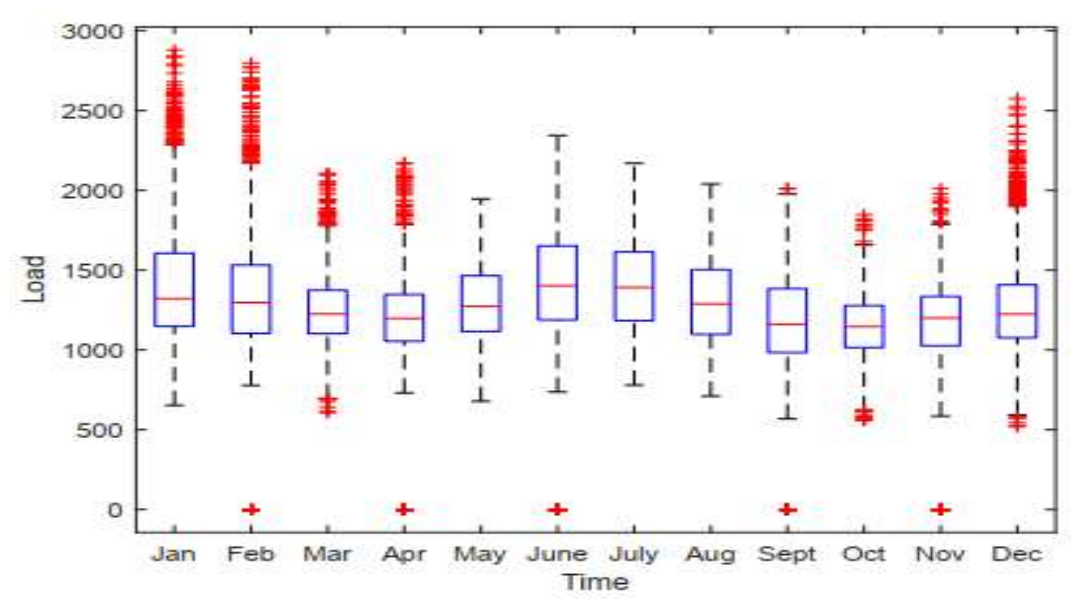

Figure 7. SA load demand 
International Journal of Advances in Scientific Research and Engineering (ijasre), Vol 6 (10), October -2020

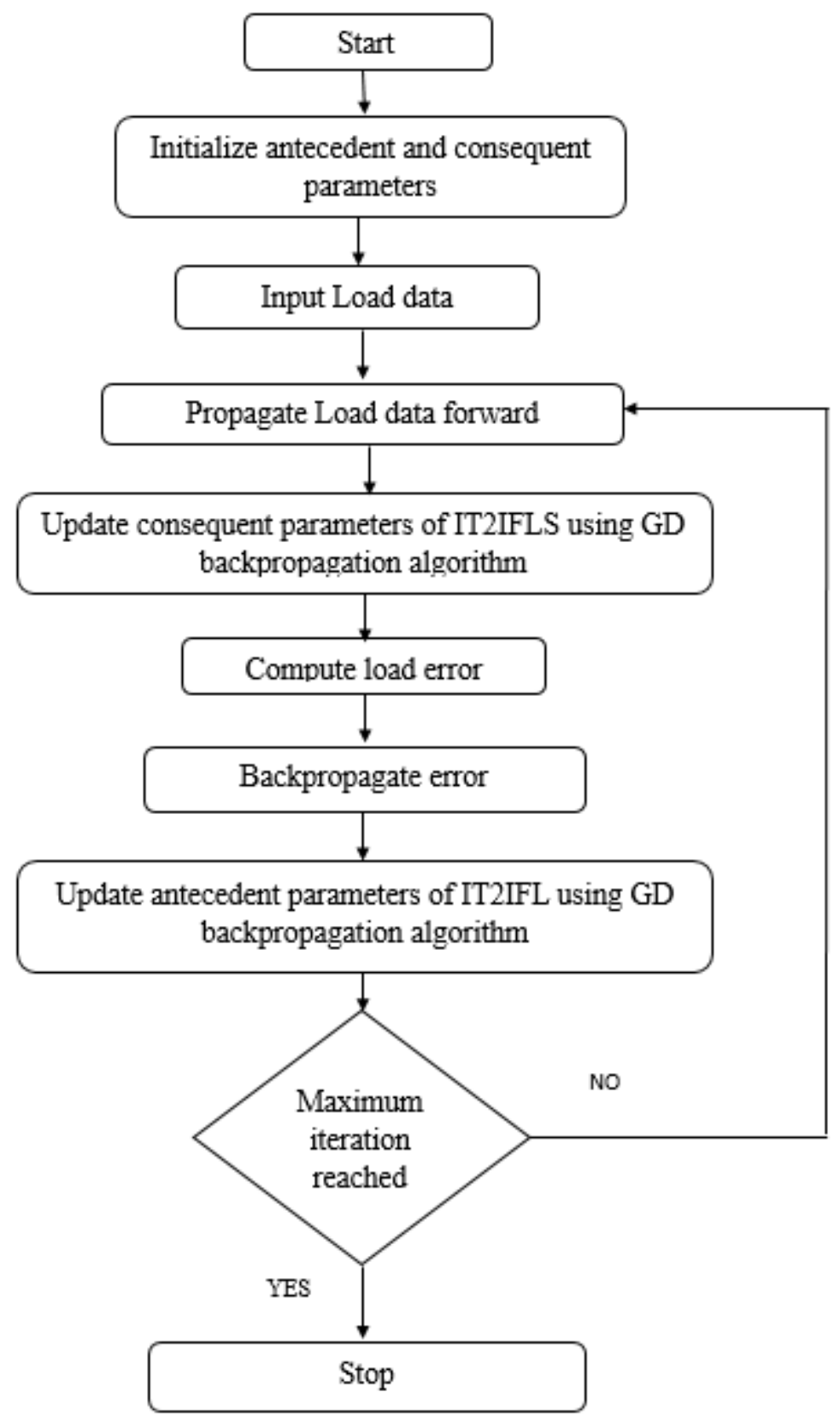

Figure 8. Flowchart of IT2IFLS load forecast

\section{PERFORMANCE EVALUATION}

The performances of IT2IFLS-VSTLF and IT2IFLS-STLF are measured using three most popular metrics for load forecasting namely: mean absolute error (MAE), symmetric mean absolute percentage error (SMAPE) and root mean squared error (RMSE) defined as:

$$
\text { MAE }=\frac{1}{T} \sum_{i=1}^{T}\left|p^{a}-p\right|
$$

where $T$ is the total number of observations.

$$
\begin{aligned}
& \mathrm{RMSE}=\sqrt{\frac{1}{T} \sum_{i=1}^{T}\left(p^{a}-p\right)^{2}} \\
& \mathrm{SMAPE}=\frac{1}{T} \sum_{i=1}^{T} \frac{\left|p^{a}-p\right|}{\left(\left|p^{a}+p\right|\right) / 2} * 100
\end{aligned}
$$




\subsection{Half-Hourly Forecasting - Very Short-Term Horizon}

For the first instance, very short-term forecasting horizon is adopted. This implies that the forecasting is on half-hourly basis. For each of the regions, the data is modelled as time series. For simplicity, we adopt the current value and four previous values. Thus, the forecasting function is represented as: $f(x(t-4) ; x(t-3) ; x(t-2) ; x(t-1) ; x(t) ; x(t+1))$ where $x(t+1)$ is the output to be predicted. For a meaningful and objective prediction, all experiments are repeated 10 times (10-fold cross validation) with 100 epochs each and the average results computed. For comparison, IT2FLS and ANN are also constructed to evaluate the performance of IT2IFLS. The ANN is adopted because it forms part of the building block of these fuzzy logic systems. The ANN is implemented in MATLAB using neural network toolbox. The hidden neuron is set to 5 with one output neuron. For a fair comparison, the GD is also adopted to tune the parameters of the ANN and IT2FLS. Shown in Table 1 to 5 are the averages of 10 runs of each model using the different performance metrics for each region. As shown in the tables, IT2IFLS performs best overall in most instances than ANN and traditional IT2FLS. However, for the VSTLF horizon, ANN exhibits improved performance in forecasting three instances than the fuzzy logic approaches in terms of SMAPE as depicted in Tables 1 and 2 . ANN performs better with reduced SMAPE during Spring in NSW-VSTLF, summer and spring seasons in VIC-VSTLF. It is interesting to also observe that in some cases such as during autumn in NSW and autumn in Victoria regions, the traditional IT2FLS with no hesitation degree outperforms hesitation-enabled IT2IFLS. This goes to show that in some cases, the simpler traditional IT2FLS may forecast better than the complex IT2IFLS.

Table 1: New South Wales VSTLF

\begin{tabular}{|l|l|c|l|l|l|l|l|l|l|}
\hline & \multicolumn{3}{|c|}{ NN } & \multicolumn{3}{c|}{ IT2FLS } & \multicolumn{3}{c|}{ IT2IFLS } \\
\hline Season & RMSE & MAE & SMAPE & RMSE & MAE & SMAPE & RMSE & MAE & SMAPE \\
\hline Summer & 0.0271 & 0.0094 & 3.1660 & 0.0098 & 0.0040 & 1.5088 & $\mathbf{0 . 0 0 8 0}$ & $\mathbf{0 . 0 0 3 2}$ & $\mathbf{1 . 1 5 4 7}$ \\
\hline Autumn & 0.0318 & 0.0205 & 5.6463 & $\mathbf{0 . 0 1 3 4}$ & $\mathbf{0 . 0 0 5 2}$ & $\mathbf{0 . 8 1 3 4}$ & 0.0137 & 0.0053 & 0.8365 \\
\hline Winter & 0.0785 & 0.0421 & 13.5096 & 0.0509 & 0.0175 & 5.4487 & $\mathbf{0 . 0 4 8 8}$ & $\mathbf{0 . 0 1 6 9}$ & $\mathbf{5 . 4 0 2 8}$ \\
\hline Spring & 0.0258 & 0.0078 & $\mathbf{1 . 4 5 3 7}$ & 0.0099 & 0.0043 & 2.3143 & $\mathbf{0 . 0 0 9 0}$ & $\mathbf{0 . 0 0 3 9}$ & 2.0115 \\
\hline
\end{tabular}

Table 2: Victoria VSTLF

\begin{tabular}{|l|l|c|l|l|c|l|c|c|l|}
\hline & \multicolumn{3}{|c|}{ NN } & \multicolumn{3}{c|}{ IT2FLS } & \multicolumn{3}{c|}{ IT2IFLS } \\
\hline & RMSE & MAE & SMAPE & RMSE & MAE & SMAPE & RMSE & MAE & SMAPE \\
\hline Summer & 0.0111 & 0.0081 & $\mathbf{1 . 6 7 1 2}$ & 0.0114 & 0.0045 & 2.0141 & $\mathbf{0 . 0 1 0 0}$ & $\mathbf{0 . 0 0 3 9}$ & 1.6787 \\
\hline Autumn & 0.0306 & 0.0200 & 5.4445 & $\mathbf{0 . 0 3 1 5}$ & $\mathbf{0 . 0 1 0 8}$ & $\mathbf{1 . 6 3 1 6}$ & 0.0320 & 0.0111 & 1.6722 \\
\hline Winter & 0.0233 & 0.0180 & 5.3939 & 0.0153 & 0.0064 & 1.9975 & $\mathbf{0 . 0 1 3 5}$ & $\mathbf{0 . 0 0 5 7}$ & $\mathbf{1 . 6 9 7 3}$ \\
\hline Spring & 0.0226 & 0.0116 & $\mathbf{1 . 9 3 0 6}$ & 0.0158 & 0.0067 & 2.7723 & $\mathbf{0 . 0 1 4 8}$ & $\mathbf{0 . 0 0 5 9}$ & 2.3999 \\
\hline
\end{tabular}

Table 3: Tasmania VSTLF

\begin{tabular}{|l|l|c|l|c|c|c|c|c|l|}
\hline & \multicolumn{3}{|c|}{ NN } & \multicolumn{3}{c|}{ IT2FLS } & \multicolumn{3}{c|}{ IT2IFLS } \\
\hline & RMSE & MAE & SMAPE & RMSE & MAE & SMAPE & RMSE & MAE & SMAPE \\
\hline Summer & 0.0331 & 0.0163 & 3.9458 & 0.0219 & 0.0088 & 1.6154 & $\mathbf{0 . 0 2 1 6}$ & $\mathbf{0 . 0 0 8 7}$ & $\mathbf{1 . 5 8 7 8}$ \\
\hline Autumn & 0.0620 & 0.0389 & 7.7347 & 0.0307 & 0.0107 & 1.3427 & $\mathbf{0 . 0 3 0 2}$ & $\mathbf{0 . 0 1 0 3}$ & $\mathbf{1 . 2 9 6 8}$ \\
\hline Winter & 1.3067 & 1.2235 & 6.0812 & 0.0183 & 0.0076 & 1.9296 & $\mathbf{0 . 0 1 8 2}$ & $\mathbf{0 . 0 0 7 5}$ & $\mathbf{1 . 9 0 7 9}$ \\
\hline Spring & 0.0204 & 0.0126 & 1.8142 & 0.0161 & 0.0066 & 1.8439 & $\mathbf{0 . 0 1 5 6}$ & $\mathbf{0 . 0 0 2 3}$ & $\mathbf{1 . 7 8 0 4}$ \\
\hline
\end{tabular}

Table 4: South Australia VSTLF

\begin{tabular}{|l|l|c|l|l|l|l|l|c|l|}
\hline & \multicolumn{4}{|c}{ NN } & \multicolumn{3}{c|}{ IT2FLS } & \multicolumn{3}{c|}{ IT2IFLS } \\
\hline & RMSE & MAE & SMAPE & RMSE & MAE & SMAPE & RMSE & MAE & SMAPE \\
\hline Summer & 0.0160 & 0.0098 & 1.9096 & 0.0126 & 0.0049 & 1.3760 & $\mathbf{0 . 0 1 1 9}$ & $\mathbf{0 . 0 0 4 5}$ & $\mathbf{1 . 2 4 2 8}$ \\
\hline Autumn & 0.0578 & 0.0387 & 9.7617 & 0.0319 & 0.0118 & 2.2789 & $\mathbf{0 . 0 3 2 1}$ & $\mathbf{0 . 0 1 1 7}$ & $\mathbf{2 . 2 7 2 6}$ \\
\hline Winter & 0.0315 & 0.0222 & 8.5386 & 0.0199 & 0.0079 & 2.7165 & $\mathbf{0 . 0 1 9 1}$ & $\mathbf{0 . 0 0 7 4}$ & $\mathbf{2 . 5 7 9 1}$ \\
\hline Spring & 0.0303 & 0.0179 & 3.3892 & 0.0195 & 0.0074 & 2.1095 & $\mathbf{0 . 0 1 8 7}$ & $\mathbf{0 . 0 0 6 9}$ & $\mathbf{1 . 9 1 8 1}$ \\
\hline
\end{tabular}


International Journal of Advances in Scientific Research and Engineering (ijasre), Vol 6 (10), October -2020

Table 5: Queensland VSTLF

\begin{tabular}{|l|c|c|l|l|c|l|c|c|l|}
\hline & \multicolumn{3}{|c|}{ NN } & \multicolumn{3}{c|}{ IT2FLS } & \multicolumn{3}{c|}{ IT2IFLS } \\
\hline & RMSE & MAE & SMAPE & RMSE & MAE & SMAPE & RMSE & MAE & SMAPE \\
\hline Summer & 0.0157 & 0.0116 & 3.4389 & 0.0131 & 0.0053 & 1.6713 & $\mathbf{0 . 0 1 1 7}$ & $\mathbf{0 . 0 0 4 5}$ & $\mathbf{1 . 3 8 3 1}$ \\
\hline Autumn & 0.0159 & 0.0120 & 5.4290 & 0.0131 & 0.0054 & 2.6580 & $\mathbf{0 . 0 1 0 5}$ & $\mathbf{0 . 0 0 4 3}$ & $\mathbf{2 . 1 3 9 0}$ \\
\hline Winter & 0.0795 & 0.0505 & 13.3140 & 0.0479 & 0.0164 & 5.4450 & $\mathbf{0 . 0 4 7 7}$ & $\mathbf{0 . 0 1 6 0}$ & $\mathbf{5 . 1 6 2 8}$ \\
\hline Spring & 0.0232 & 0.0159 & 3.9781 & 0.0153 & 0.0060 & 1.4829 & $\mathbf{0 . 0 1 7 6}$ & $\mathbf{0 . 0 0 5 8}$ & $\mathbf{1 . 3 5 5 3}$ \\
\hline
\end{tabular}

\subsection{A Day Ahead Forecasting - Short Term Forecasting Horizon}

Next, the different datasets are analyzed based on short term forecasting horizon i.e. one-day ahead load forecasting. The input generating function is given as: $f(x(t-4) ; x(t-3) ; x(t-2) ; x(t-1) ; x(t) ; x(t+48))$ where $x(t+48)$ is the output to be predicted. Similar to VSTLF, 10 runs of the experiments are conducted and the average computed. The performance of IT2IFLS is compared with those of ANN and traditional IT2FLS and the results presented in Tables 6 to 10. As indicated in the tables, IT2IFLS outperforms traditional models such as ANN and IT2FLS in almost all the instances during the STLF. This is an indication that the presence of non-membership functions and hesitation indices provide IT2IFLS the capacity to adequately predict the load as closely as possible to the real values. Interestingly, it is observed that with increasing time horizon, IT2IFLS tends to be more robust than the traditional IT2FLS in these forecasting instances.

Table 6: New South Wales STLF

\begin{tabular}{|l|l|l|l|l|l|l|l|l|l|}
\hline & \multicolumn{3}{|c|}{ NN } & \multicolumn{3}{c|}{ IT2FLS } & \multicolumn{3}{c|}{ IT2IFLS } \\
\hline & RMSE & MAE & SMAPE & RMSE & MAE & SMAPE & RMSE & MAE & SMAPE \\
\hline Summer & 0.1074 & 0.0785 & 20.0768 & 0.0769 & 0.0342 & 6.254 & $\mathbf{0 . 0 4 8 1}$ & $\mathbf{0 . 0 2 1 3}$ & $\mathbf{2 . 7 2 8 3}$ \\
\hline Autumn & 0.0929 & 0.0699 & 17.1823 & 0.0461 & 0.0185 & 5.2890 & $\mathbf{0 . 0 4 4 0}$ & $\mathbf{0 . 0 1 8 2}$ & $\mathbf{5 . 2 4 1 7}$ \\
\hline Winter & 0.1259 & 0.0939 & 21.9694 & 0.0861 & 0.0368 & 14.1792 & $\mathbf{0 . 0 7 5 5}$ & $\mathbf{0 . 0 3 0 4}$ & $\mathbf{9 . 8 1 7 2}$ \\
\hline Spring & 0.0597 & 0.0480 & 18.2380 & 0.0663 & 0.0315 & 18.351 & $\mathbf{0 . 0 4 5 8}$ & $\mathbf{0 . 0 3 3 2}$ & $\mathbf{1 7 . 2 1 5 3}$ \\
\hline
\end{tabular}

Table 7: Victoria STLF

\begin{tabular}{|l|l|c|l|l|l|l|l|l|l|}
\hline & \multicolumn{3}{|c|}{ NN } & \multicolumn{3}{c|}{ IT2FLS } & \multicolumn{3}{c|}{ IT2IFLS } \\
\hline & RMSE & MAE & SMAPE & RMSE & MAE & SMAPE & RMSE & MAE & SMAPE \\
\hline Summer & 0.1113 & 0.0811 & 26.2023 & 0.0699 & 0.0255 & 8.8068 & $\mathbf{0 . 0 5 0 5}$ & $\mathbf{0 . 0 2 0 3}$ & $\mathbf{4 . 3 9 4 7}$ \\
\hline Autumn & 0.1602 & 0.1268 & 23.7029 & 0.0776 & 0.0306 & 7.4369 & $\mathbf{0 . 0 7 5 9}$ & $\mathbf{0 . 0 3 0 1}$ & $\mathbf{7 . 2 1 0 7}$ \\
\hline Winter & 0.1308 & 0.0995 & 19.0669 & 0.1331 & 0.0631 & 17.5891 & $\mathbf{0 . 1 0 2 0}$ & $\mathbf{0 . 0 4 5 2}$ & $\mathbf{1 3 . 7 9 5 5}$ \\
\hline Spring & 0.0719 & 0.0527 & 7.9712 & 0.0383 & 0.0155 & 2.3040 & $\mathbf{0 . 0 3 6 9}$ & $\mathbf{0 . 0 1 5 0}$ & $\mathbf{2 . 2 3 1 4}$ \\
\hline
\end{tabular}

Table 8: Tasmania STLF

\begin{tabular}{|l|l|c|l|l|l|l|l|c|l|}
\hline & \multicolumn{3}{|c|}{ NN } & \multicolumn{3}{c|}{ IT2FLS } & \multicolumn{3}{c|}{ IT2IFLS } \\
\hline & RMSE & MAE & SMAPE & RMSE & MAE & SMAPE & RMSE & MAE & SMAPE \\
\hline Summer & 0.1555 & 0.1252 & 20.6736 & 0.0769 & 0.0343 & 6.2540 & $\mathbf{0 . 0 4 8 1}$ & $\mathbf{0 . 0 2 1 3}$ & $\mathbf{2 . 7 2 8 3}$ \\
\hline Autumn & 0.1318 & 0.1017 & 19.3636 & 0.0758 & 0.0325 & 6.8223 & $\mathbf{0 . 0 6 9 3}$ & $\mathbf{0 . 0 2 9 4}$ & $\mathbf{6 . 0 6 4 0}$ \\
\hline Winter & 0.1315 & 0.1056 & 20.8551 & 0.0999 & 0.0434 & 11.7267 & $\mathbf{0 . 0 8 9 0}$ & $\mathbf{0 . 0 3 7 5}$ & $\mathbf{9 . 9 0 3 0}$ \\
\hline Spring & 0.0581 & 0.0446 & 6.2066 & 0.0260 & 0.0106 & 1.5087 & $\mathbf{0 . 0 2 4 5}$ & $\mathbf{0 . 0 1 0 0}$ & $\mathbf{1 . 4 3 4 8}$ \\
\hline
\end{tabular}

Table 9: South Australia STLF

\begin{tabular}{|l|l|c|l|l|c|l|l|l|l|}
\hline & \multicolumn{3}{|c|}{ NN } & \multicolumn{3}{c|}{ IT2FLS } & \multicolumn{3}{c|}{ IT2IFLS } \\
\hline & RMSE & MAE & SMAPE & RMSE & MAE & SMAPE & RMSE & MAE & \multicolumn{1}{c|}{ SMAPE } \\
\hline Summer & 0.1213 & 0.0927 & 24.9012 & 0.1042 & 0.0402 & 10.7641 & $\mathbf{0 . 0 8 8 3}$ & $\mathbf{0 . 0 3 4 4}$ & $\mathbf{7 . 6 7 2 6}$ \\
\hline Autumn & 0.1137 & 0.0886 & 18.8126 & 0.0769 & 0.0353 & 8.8699 & $\mathbf{0 . 0 7 2 9}$ & $\mathbf{0 . 0 3 3 1}$ & $\mathbf{8 . 3 8 2 3}$ \\
\hline Winter & 0.1221 & 0.0999 & 22.8535 & 0.0908 & 0.0404 & 14.0457 & $\mathbf{0 . 0 9 0 6}$ & $\mathbf{0 . 0 3 9 7}$ & $\mathbf{1 2 . 3 3 2 2}$ \\
\hline Spring & 0.0919 & 0.0683 & 11.2956 & $\mathbf{0 . 0 4 8 1}$ & $\mathbf{0 . 0 2 0 0}$ & $\mathbf{3 . 5 4 3 3}$ & 0.0571 & 0.0252 & 4.3078 \\
\hline
\end{tabular}


International Journal of Advances in Scientific Research and Engineering (ijasre), Vol 6 (10), October -2020

Table 10: Queensland STLF

\begin{tabular}{|l|l|c|l|l|l|l|l|l|l|}
\hline & \multicolumn{3}{|c|}{ NN } & \multicolumn{3}{c|}{ IT2FLS } & \multicolumn{3}{c|}{ IT2IFLS } \\
\hline & RMSE & MAE & SMAPE & RMSE & MAE & SMAPE & RMSE & MAE & SMAPE \\
\hline Summer & 0.1093 & 0.0835 & 19.7968 & 0.0704 & 0.0276 & 8.1470 & $\mathbf{0 . 0 6 1 5}$ & $\mathbf{0 . 0 2 7 2}$ & $\mathbf{6 . 7 1 7 5}$ \\
\hline Autumn & 0.0862 & 0.0707 & 18.2019 & 0.0394 & 0.0147 & 6.8274 & $\mathbf{0 . 0 3 8 9}$ & $\mathbf{0 . 0 1 4 6}$ & $\mathbf{6 . 6 6 9 1}$ \\
\hline Winter & 0.1139 & 0.0867 & 19.7725 & 0.0917 & 0.0411 & 19.9580 & $\mathbf{0 . 0 7 5 8}$ & $\mathbf{0 . 0 3 0 5}$ & $\mathbf{9 . 5 7 9 2}$ \\
\hline Spring & 0.1141 & 0.0841 & 20.5519 & 0.0639 & 0.0267 & 7.8141 & $\mathbf{0 . 0 6 3 7}$ & $\mathbf{0 . 0 2 1 8}$ & $\mathbf{6 . 7 2 5 4}$ \\
\hline
\end{tabular}

\section{CONCLUSION}

This study presents the analysis of load forecasting for the first time using IT2IFLS. Electric load data in the year 2018 obtained from AEMO are investigated. For objective evaluation of the model, comparison is made with traditional models such as ANN and IT2FLS on the same problem instances. From the tables, it is shown that the prediction results of IT2FLS tend to be close to those of IT2IFLS while IT2FLS performed better in very few instances. However, in the overall, IT2IFLS with membership functions, non-membership functions that are fuzzy and with hesitation indices outperforms both ANN and IT2FLS with reduced RMSE, MAE and SMAPE. We conjectured that the integration of the non-membership functions provides IT2IFLS with additional degrees of freedom to model uncertainty in electricity load better than the traditional IT2FLS. In addition, the hesitation indices bring the representation very close to the everyday human language and reasoning with improved accuracy. It is also demonstrated here that with increasing forecasting time scale, IT2IFLS becomes more robust than the traditional IT2FLS and ANN. In the future, we intend to explore the general IT2IFLS with other learning algorithms such as particle swarm optimization, simulated annealing and extended Kalman filter.

\section{REFERENCE}

[1] S. Narjes Fallah, M. Ganjkhani, S. Shamshirband and K. Chau, "Computational Intelligence on Short-Term Load Forecasting: A Methodological Overview”, Energies, 2019, 12, 393; doi:10.3390/en12030393.

[2] D. Ali, M. Yohanna, M. I. Puwu and B. M. Garkida, "Long-term load forecast modelling using a fuzzy logic approach". Pacific Science Review A: Natural Science and Engineering, 2016, 18(2), 123-127.

[3] E. Akarslan, and F. O. Hocaoglu, "A novel short-term load forecasting approach using Adaptive Neuro-Fuzzy Inference System". In 2018 6th International Istanbul Smart Grids and Cities Congress and Fair (ICSG) April 2018, pp. 160-163. IEEE.

[4] H. Javedani Sadaei, F. Gadelha Guimares, C. Jos da Silva, M. Hisyam Lee, T. Eslami, "Short-term load forecasting method based on fuzzy time series, seasonality and long memory process", Int. J. Approx. Reason. 2017, http://dx.doi.org/10.1016/j.ijar.2017.01.006.

[5] E. Mocanu, P. H. Nguyen, M. Gibescu, W. L. Kling, "Deep learning for estimating building energy consumption", Sustainable Energy, Grids and Networks 6 (2016) 9199.

[6] F. Castro Torrini, R. Castro Souza, F. Luiz Cyrino Oliveira, J. Francisco Moreira Pessanha, "Long term electricity consumption forecast in Brazil: A fuzzy logic approach", Socio-Economic Planning Sciences (2016), doi: 10.1016/j.seps.2015.12.002.

[7] M. R. Haq and Z. Ni, “A New Hybrid Model for Short-Term Electricity Load Forecasting”.IEEE Access, 2019, 7, 125413-125423.

H. Zheng, J. Yuan, and L. Chen, "Short-term load forecasting using EMD-LSTM neural networks with a Xgboost algorithm for feature importance evaluation”. Energies, 2017, 10(8), 1168. 
[10] M. A. Al Amin, and M. A. Hoque, "Comparison of ARIMA and SVM for Short-term Load Forecasting”. In 9th Annual Information Technology, Electromechanical Engineering and Microelectronics Conference (IEMECON), March 2019, pp. 1-6. IEEE.

[11] Y. Ma, Q. Zhang, J. Ding, Q. Wang, and J. Ma, “Short Term Load Forecasting Based on iForest-LSTM". In 14th IEEE Conference on Industrial Electronics and Applications (ICIEA), June 2019, pp. 2278-2282. IEEE.

[12] T. Ouyang, Y. He, H. Li, Z. Sun, and S. Baek, "Modeling and forecasting short-term power load with copula model and deep belief network". IEEE Transactions on Emerging Topics in Computational Intelligence, 2019, 3(2), $127-136$.

[13] G. Yan, T. Han, W. Zhang, and S. Zhao, "Short-Term Load Forecasting of Smart Grid Based on Load Spatial-Temporal Distribution”. In 2019 IEEE Innovative Smart Grid Technologies-Asia (ISGT Asia), May 2019, pp. 781-785. IEEE.

[14] K. Chen, K. Chen, Q. Wang, Z. He, J. Hu, and J. He, "Short term load forecasting with deep residual networks", IEEE Transactions on Smart Grid, 2018, 10(4), 3943-3952.

[15] G. D. Merkel, R. J. Povinelli and R. H. Brown, "Short-term load forecasting of natural gas with deep neural network regression". Energies, 11(8), 2018.

[16] S. Ryu, J. Noh and H. Kim, "Deep neural network based demand side short term load forecasting”. Energies, 2017, 10(1), 3 .

[17] A. Khosravi, S. Nahavandi, D. Creighton and D. Srinivasan, "Interval type-2 fuzzy logic systems for load forecasting: A comparative study”. IEEE Transactions on Power Systems, 2012, 27(3), 1274-1282.

[18] L. A. Zadeh, Fuzzy sets. Information and control, 1965, 8(3), 338-353.

[19] G. Sideratosa, A. Ikonomopoulosa, N. D. Hatziargyriou, “A novel fuzzy-based ensemble model for load forecasting using hybrid deep neural networks, Electric Power Systems Research 178, 2020, 106025.

[20] C. Li, M. Tang, G. Zhang, R. Wang and C. Tian, "A hybrid short term building electrical load forecasting model combining the periodic pattern, fuzzy system, and wavelet transform". International Journal of Fuzzy Systems, 2020 , 22(1), 156-171.

[21] D. K. Chaturvedi, A. P. Sinha, and O. P. Malik, "Short term load forecast using fuzzy logic and wavelet transform integrated generalized neural network". International Journal of Electrical Power and Energy Systems, 2015, 67, 230237.

[22] Cevik, H. H., and Cunka, M. (2015). Short-term load forecasting using fuzzy logic and ANFIS. Neural Computing and Applications, 26(6), 1355-1367.

[23] L. A. Zadeh, "The concept of a linguistic variable and its application to approximate reasoningII." Information sciences, $1975,8(4), 301-357$.

[24] U. Umoh, I. Umoeka, M. Ntekop and E. Babalola, "Interval type-2 fuzzy neural networks for short-term electric load forecasting: A comparative study”, International Journal on Soft Computing (IJSC), 2018, Vol.9, No.1.

[25] I. Eyoh, R. John, and G. De Maere, "Interval type-2 A-intuitionistic fuzzy logic for regression problems". IEEE Transactions on Fuzzy Systems, 2017, 26(4), 2396-2408.

[26] K.T. Atanassov, “Intuitionistic fuzzy sets”. Fuzzy Sets and Systems 20(1), 8796 (1986).

[27] I. Eyoh, R. John, and G. De Maere, "Interval type-2 intuitionistic fuzzy logic system for non-linear system prediction". In 2016 IEEE International Conference on Systems, Man, and Cybernetics (SMC) October 2016, pp. 001063-001068. IEEE. 
International Journal of Advances in Scientific Research and Engineering (ijasre), Vol 6 (10), October -2020

[28] L. Zhao, Z. Xie and Z. Zhao, "Interval Type-2 Intuitionistic Fuzzy Random Vector Functional Link Neural Network Based Spatio-temporal Prediction of Nitrogen Dioxide Concentration in Air".

[29] I. Eyoh, J. Eyoh, and R. Kalawsky, "Interval type-2 intuitionistic fuzzy logic system for time series and identification problems-a comparative study". International Journal of Fuzzy Logic Systems (IJFLS) Vol.10, No.1, DOI: 10.5121/ijfls.2020.101011.

[30] D. K. Jana, "Novel arithmetic operations on type-2 intuitionistic fuzzy and its applications to transportation problem". Pacific Science Review A: Natural Science and Engineering, 2016, 18(3), 178-189.

[31] D. D. Nguyen, L. T. Ngo, and L. T. Pham, "Interval type-2 fuzzy c-means clustering using intuitionistic fuzzy sets". In 2013 Third World Congress on Information and Communication Technologies (WICT 2013), December 2013, pp. 299304. IEEE.

[32] I. Eyoh, R. John, and G. De Maere, "Time series forecasting with interval type-2 intuitionistic fuzzy logic systems. In 2017 IEEE International Conference on Fuzzy Systems (FUZZ-IEEE), July 2017, pp. 1-6. IEEE.

[33] C. Luo, C. Tan, X. Wang, and Y. Zheng, "An evolving recurrent interval type-2 intuitionistic fuzzy neural network for online learning and time series prediction”. Applied Soft Computing, 2019, 78, 150-163.

[34] F. Zhao, Y. Chen, H. Liu, and J. Fan, "Alternate PSO-based adaptive interval type-2 intuitionistic fuzzy C-means clustering algorithm for color image segmentation. IEEE Access, 2019, 7, 64028-64039.

[35] W. Yuan, and L. Chao, “Online Evolving Interval Type-2 Intuitionistic Fuzzy LSTMNeural Networks for Regression Problems”. IEEE Access, 2019, 7, 35544-35555.

[36] I, Eyoh, U. Umoh, U. Inyang and J. Eyoh, "Derivative-based Learning of Interval Type-2 Intuitionistic Fuzzy Logic Systems for Noisy Regression Problems”, International Journal of Fuzzy Systems. 2020, 22(3), 10071019. DOI: $10.1007 / \mathrm{s} 40815-020-00806-\mathrm{z}$.

[37] K. Tai, A.-R. El-Sayed, M. Biglarbegian, C. I. Gonzalez, O. Castillo, and S. Mahmud, "Review of recent type-2 fuzzy controller applications," Algorithms, vol. 9, no. 2, p. 39, 2016.

[38] M. B. Begian, W. W. Melek, and J. M. Mendel, "Parametric design of stable type-2 tsk fuzzy systems," in IEEE Annual Meeting of the North American Fuzzy Information Processing Society, (NAFIPS), pp. 1-6, 2008.

[39] M. Nie and W. W. Tan, "Towards an efficient type-reduction method for interval type-2 fuzzy logic Systems", in Fuzzy Systems, 2008. FUZZ-IEEE 2008. (IEEE World Congress on Computational Intelligence). IEEE International Conference on, pp. 1425-1432, IEEE, 2008.

[40] D. Wu and W. W. Tan, "Computationally efficient type-reduction strategies for a type-2 fuzzy logic Controller", in Fuzzy Systems, 2005. FUZZ’05. The 14th IEEE International Conference on, pp. 353-358, IEEE, 2005. 